\title{
Faces attract infants' attention in complex displays
} Athina Andravizou*1, Teodora Gliga ${ }^{2}$, Mayada Elsabbagh ${ }^{2}$ and Mark Johnson ${ }^{2}$ \author{
and Cognitive Development, Birkbeck College, London, UK \\ * Corresponding author \\ from International Society on Brain and Behaviour: 3rd International Congress on Brain and Behaviour \\ Thessaloniki, Greece. 28 November - 2 December 2007 \\ Published: 17 April 2008 \\ Annals of General Psychiatry 2008, 7(Suppl I):S276 doi:I0.I 186/I744-859X-7-SI-S276
}

Address: ${ }^{1}$ Department of Psychology, University College London-Birkbeck College, London, UK and ${ }^{2}$ Department of Psychology, Centre for Brain

This abstract is available from: http://www.annals-general-psychiatry.com/content/7/SI/S276

(C) 2008 Andravizou et al.; licensee BioMed Central Ltd.

\section{Background}

Hersler and Hochstein [1] found in adult experiments that face "pop-out", that is they are looked at earlier and for longer, when presented together with a variety of different objects.

\section{Materials and methods}

In contrast to "classical" "pop out" studies we don't vary the number of distractors. We explored this effect in 6month old infants. Twelve slides were presented to infants, each one including one human face and five different object distractors. The objects are similar to faces regarding their shape, colour, luminance and familiarity. Also, half of the faces have direct gaze, the other averted.

\section{Results}

Results indicate that faces "pop out" among distractors. Direct and averted gaze do not differ from each other regarding pop-out effects.

\section{Conclusions}

The implications of these results are discussed.

\section{Acknowledgements}

I wish to thank Prof Mark Johnson, Dr Teodora Gliga, Dr Mayada Elsabbach all of whom helped me in the experiment, and Dr Kyrana Tsapkini, who although far away, has been very supportive to me during this year.

\section{References}

I. Hershler O, Hochstein S: At first sight: A high-level pop out effect for faces. Vision Research 2005, 45: 1707-I724.
2. Farroni T, Csibra G, Simion F, Johnson MH: Eye contact detection in humans from birth. Proc Natl Acad Sci USA 2002, 99:9602-9605. 3. Johnson M, Dziurawiec S, Ellis H, Morton J: Newborns preferential tracking of face-like stimuli and its' subsequent decline. Cognition 1991, 40:1-19. 